\title{
Automatic Grading of Appearance Retention of Carpets Using Intensity and Range Images
}

\author{
S. A. Orjuela ${ }^{\text {a,d }}$, B. Ortiz-Jaramillo ${ }^{b}$, E. Vansteenkiste ${ }^{a}$, F. Rooms ${ }^{\text {a }}$, S. De \\ Meulemeester $^{\text {b }}$, R. de Keyser ${ }^{c}$, L. Van Langenhove ${ }^{b}$ and W. Philips ${ }^{a}$

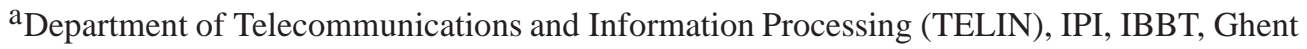 \\ University, Belgium \\ ${ }^{\mathrm{b}}$ Department of Textiles, Ghent University, Belgium \\ ${ }^{\mathrm{c}}$ Department of Electrical energy, Systems and Automation, Ghent University, Belgium \\ d Antonio Nariño University, Colombia
}

\begin{abstract}
Textiles are mainly used for decoration and protection. In both cases, original appearance and its retention are important factors for customers. Therefore, evaluation of appearance parameters are critical for quality assurance purposes, while and after manufacturing, to determine lifetime and/or beauty of textile products. Particularly, appearance retention of textile products is commonly certified with grades, which are currently assigned by human experts. However, manufacturers would prefer a more objective system. In this paper we present an objective system for grading appearance retention, particularly, for textile floor coverings. Changes in appearance are quantified by using linear regression models on texture features extracted from intensity and range images. Range images are obtained by our own laser scanner, reconstructing the carpet surface using two methods that have been previously presented. We extract texture features using a variant of the local binary pattern technique based on detecting those patterns whose frequencies are related to the appearance retention grades. We test models for eight types of carpets. Results show that the proposed approach describes the degree of wear with a precision within the range allowed to human inspectors by international standards. The methodology followed in this experiment has been designed to be general for evaluating global deviation of texture in other types of textiles as well as other surface materials.
\end{abstract}

Keywords: LBP techniques, Image Analysis, Wear Analysis, Texture Analysis, Appearance Retention 


\section{INTRODUCTION}

Nowadays, the textile floor cover industry offers a wide range of carpets, of which those that retain their original appearance for longer periods are preferred by end customers. The Appearance Retention (AR) in carpets is validated and certified following quality standards based on simulating traffic exposure [1]. There are several standardized tests to provide Euronorm (EN) and International Standards Organization (ISO) certification of AR in carpets [2]. Particularly, different levels of traffic exposure are simulated, e.g. by degrading surfaces of new carpet samples (accelerating the wear) with mechanical devices during different periods of time [3]. After simulating traffic exposure a carpet sample is called a fatigued specimen and its degree of change is expressed by using a single overall AR grade [4]. The overall change is determined by independently evaluating changes in pattern, thickness, hairiness, color and texture. These visual characteristics are numerically expressed and then combined to calculate the overall AR grade.

The change in appearance of a fatigued specimen is evaluated by comparing changes with respect to the original appearance of an unfatigued specimen and certified AR references. AR references are a set of fatigued specimen samples representing different AR grades. It is expensive and time consuming to compose an AR quality standard reference. Besides, features of physical fatigued specimen samples change over time and are exposed to involuntary detriment. Therefore, some certifications use photographs of the fatigued specimens instead. Particularly, the AR references in Europe are composed of physical samples of fatigued specimens while in America the AR references are composed of photographs.

Currently, the AR retention in carpets is evaluated by at least three certified experts, which is hampered by human subjectivity. The human assessment results in inconsistencies between experts with errors up to a half grade in the AR grades [4]. The main drawbacks for this system are that it is time consuming, prompt to human errors and it requires at least three experts, who are not always available, especially within small companies.

Several studies have been performed in search of an objective AR grading assessment using image analysis techniques [5-12]. Most of the research has been conducted using gray-scale images, which require less rigorous control of illumination and camera characteristics than color images. Tested algorithms include gray value histogram analysis, co-ocurrence matrices, local intensity variation filters, statistical measures and edge detection filters. Results of some 
of these algorithms show successful systems with correct assessment over 95\% developed with specific algorithms for limited carpet types [11,12]. None of these methods succeed considering diverse carpet types.

Recently, we proposed an algorithm based on the Local Binary Pattern (LBP) technique to appropriately quantify the degree of wear [13]. We eill briefly explain this algorithm in Section 2.2. We tested the algorithm using certified photographs for the American standard and photographs taken under fixed luminance conditions for the European standard. Although the method correctly distinguished AR grades in certified photographs, we found that non certified photographs are non-robust enough for describing the appearance changes of carpets [14].

Several researchers have also explored the use of depth images, reflecting 3D structure of the carpets. This is an important feature evaluated by experts, which is not correctly acquired in photographs. Recently, we presented a scanner specifically designed for scanning carpets using structured light triangulation [15]. These type of scanners have the advantage of low color sensitivity compared to other 3D imaging methods [16-18]. The depth information is digitized into a range image, where the pixels of the image represent depth. This type of scanners has previously been used to characterize the 3D surface roughness of fabrics [19-22]. The performance of classical scanners has been improved by placing the carpet on a drum, which results in better capturing of the piles defining the structure of the carpet. We have proposed an automatic grading system based on image analysis algorithms using images obtained from the scanner based on a drum together with photographs [23]. Linear models for quantifying changes in appearance of fatigued specimens are computed by combining texture features based on local binary patterns from both intensity and range images. The method was appropriate for automatically grading a specific type of, i.e. loop pile carpets.

The current paper presents for the first time an automatic grading system that is already generic for carpets with low pile construction and without color patterns. Some parts of the method have been explained in detail in previous papers [13-15, 23-26]. In this paper we present an optimal combination of the previous findings. This combination significantly improves the performance of the method. The initial method has been improved by including two new components. The first new component consists of constructing the range images by applying an edge detection method based on the Gaussian pyramid representation [25]. This permits a more precisely reconstruction of the carpet surfaces. The second component consists 
of linearly modeling texture features based on local binary patterns whose probabilities of occurrence monotonically change with the AR grades [26]. The linear models computed with the new method predict the degree of wear better than those computed in previous approaches.

We tested the automatic grading system on AR references from the European standard. One linear model for each reference is computed by combining texture features based on local binary patterns extracted from both range and intensity images. The AR grade of a fatigued specimen sample within the evaluated AR references is calculated using the corresponding linear model. The paper is organized as follows. In Section 2.1, we describe the details of our proposed scanner and the carpet batch to be analysed. In Section 2.2, we describe the technique for extracting texture features. In Section 2.3 we explain how to use the texture features to estimate the AR grades for new carpet samples by using linear regression models. In Section 3, we describe the experiment, report the results and discuss the findings. Finally, in Section 4 the conclusions are drawn.

\section{MATERIALS AND METHODS}

We propose an automatic grading system in which visual characteristics related to appearance changes are independently quantified and then combined into a linear model to assess the AR grade of the fatigued specimen. We present in this paper the results of a first approach modeling only texture features extracted from intensity and depth images.

International standards are independently defined for color and appearance degradation, where appearance retention refers to evaluation of degradation on the carpet texture surfaces independently from colors. Therefore, we use texture features decoupled from colors quantifying the difference, for both types of images, between the worn and original appearance. The method is illustrated in Figure 1. The steps are explained in detail in the followings. 


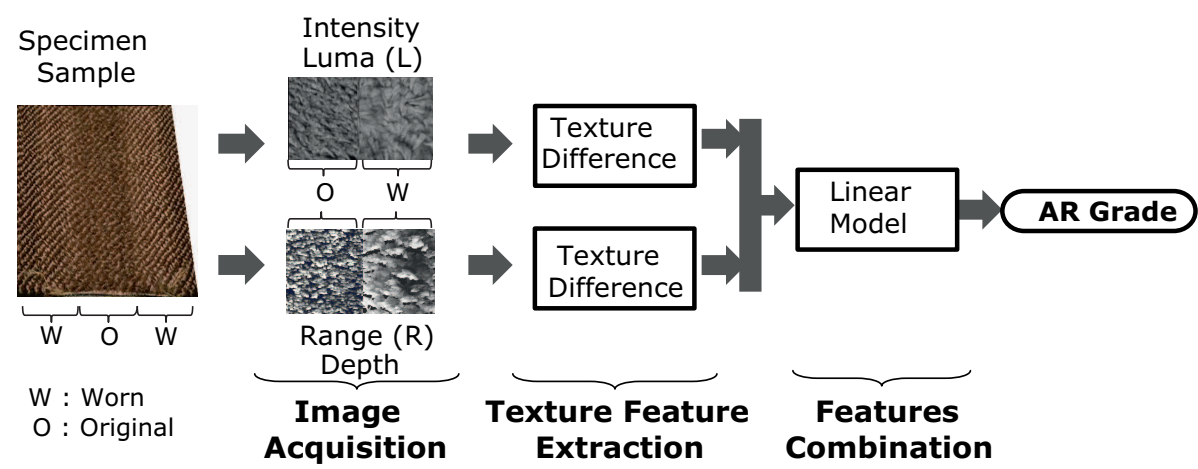

Fig. 1. Proposed method for automatic grading of appearance retention in carpets. 


\subsection{Image Acquisition}

The AR references used in our research follows the EN1471 European standard [4]. Each fatigued specimen sample contains both the appearance changes in the middle and the original appearance at both sides. The appearance changes are assessed with labels in the range from 1.0 to 5.0, with steps of half a point, where a severe change is labeled with an AR grade of 1 and original appearance with AR 5.0. The fatigued specimen samples have been collected from the following carpet types: high/low loop, loop, cut/loop, cut pile, cut design, frisé and two types of shaggy. With these fatigued specimen samples, a database consisting of both intensity and range images has been composed [14].

The database of intensity images is composed of photographs with a size of $720 \times 576$ pixels corresponding to $17.28 \times 13.82 \mathrm{~cm}^{2}$ of the surface. Thus, one pixel represents a square with size of $0.24 \mathrm{~mm}$ by $0.24 \mathrm{~mm}$. Photographs are acquired in the RGB color space. However, since we are interested in evaluating texture change independently from color change, images must be transformed to other color representation space that permits to decouple the intensity information from the colors [27]. This is possible by using the luminance component from color representations such as the YUV (component Y) or the HSV (component V) color spaces. Previous investigations evaluating the luminance component for texture analysis tasks reported non-significant difference between both color spaces [28,29]. Therefore, we can chose the YUV representation space for conducting this investigation.

The samples were additionally scanned with our carpet scanner to compose the database of range images. To scan, a fatigued specimen sample is first clamped with elastic bands upon an inox-drum. Then, a line laser generator projects a uniform line on the surface of the sample. The reflected light, containing the depth information, is captured with a 3CCD camera, configured to horizontally cover a similar surface area with the same pixel resolution as covered with the photographs. Afterwards, the drum is rotated at a controlled speed to capture frames in which the projected light is spaced at $0.24 \mathrm{~mm}$. In each frame, the reflected light due to the projection of the laser line in the carpet surface is represented with pixels as shown in Figure 2 a). One pixel per column is used for representing the depth of the surface with one array per frame as shown in Figure 2 b). Finally, a range image, with the same pixel resolution as in the photographs, is constructed by sequentially aligning the arrays. The arrays are obtained using two approaches: the RefLight and the EdgeWav methods [25]. We briefly summarize these methods below. 
A) The RefLight method. With this method the array is obtained by detecting the highest position of the highest intensity value on each column of the frame [15]. E.g. The brightest pixel in column 4 of Figure 2 a) is located at row 92 . When the highest intensity value is located in more than one row, the top location is chosen as in column 15 of Figure 2 a, where row 94 is selected. The Reflight method characterizes the texture given by a carpet surface without separating specific details such as distorted fibers from the base of the structure. 
a)

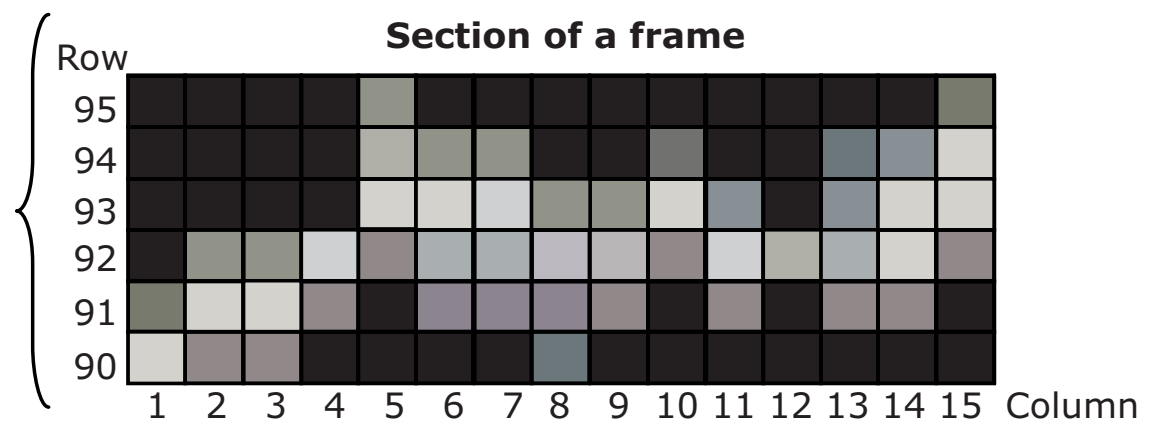

b) \{

Array

Fig. 2. The RefLight method. a) The Figure displays a section of a frame from rows 90 to 95 and columns 1 to 15 . The reflection of the laser line is represented with pixels there light colors correspond to bright reflection. b) Array obtained with the RefLight method to identify the relevant depth values in the frame section. Each value in the array represents the row where the highest intensity values are located at a highest position per column. 
B) The EdgeWav method. The EdgeWave method has been designed for characterizing texture due only to the base surface structure. The method consists in detecting at different scales the edges corresponding to tuft tips and then merging them into one image [30]. Edges are detected in each scale by using a Sobel operator. Experimentally, edges of tuft tips can be visually distinguished up to a third resolution level. The edges in each scale are binarized using a threshold to separate tuft tips from noise and distorted fibres. This threshold is calculated using the Lipschitz exponent, which expresses the local regularity of a neighbourhood [31]. The Lipschitz exponent is small where only fine texture or noise are present and large where smoother features or continuous edges are present. The resulting binary edges are merged into one image by using the logical OR operation. The average in terms of the locations of the pixels corresponding to edges is computed to obtain only one value of depth per column in the merged image. The method is graphically illustrated in Figure 3. 


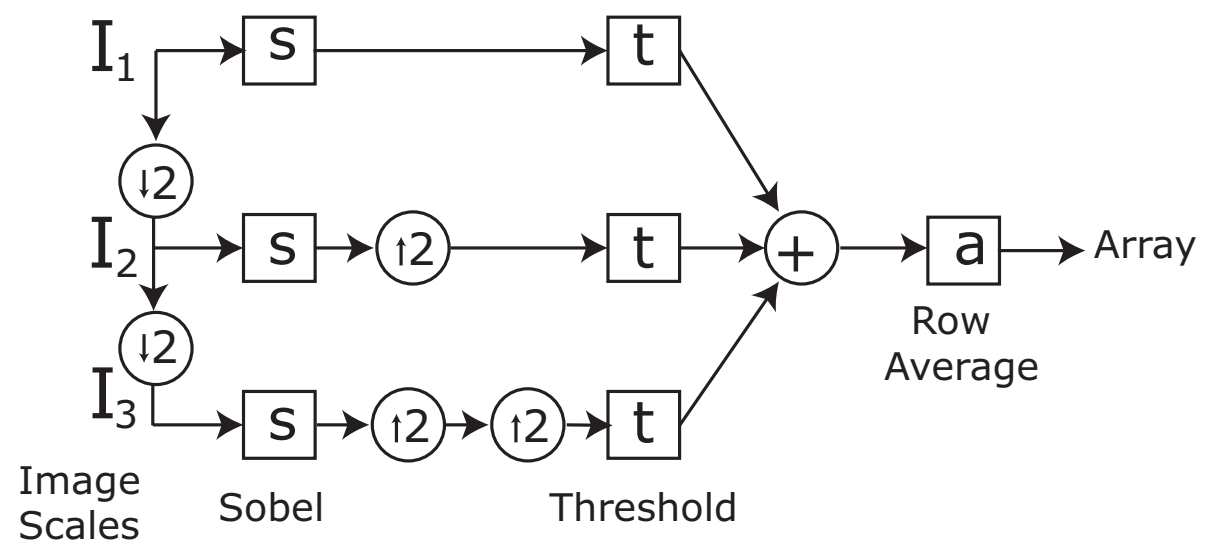

Fig. 3. The EdgeWav method. Edges are detected with a Sobel (s) operator for three consecutive scales, given by Images $I_{1}, I_{2}$ and $I_{3}$. Tuft tips are separated from both noise and distorted fibres by thresholding $(\mathrm{t})$ the images using the Lipschitz exponent. Binary edges are merged into one image using the or operation, indicated with the symbol + . The symbol $2 \downarrow$ refers to Gaussian downscaling and the symbol $2 \uparrow$ to upscaling. The average (a) in terms of row positions of the pixels corresponding to edges is computed to obtain only one value of depth per column in the merged image. 
The RefLight method implicitly contains information about hairiness since this method delineates distorted fibers as opposed to the capturing base of the carpet structure such as the WavEdge method. Therefore, both methods are complementary, thus combining both leads to better results as will be shown in Section 3.

We covered the entire surface for each AR grade by capturing 5 pairs of images corresponding to worn and original appearance. Therefore, each AR grade in this experiment is associated with a set of 30 images, (ten images for each method). All images have the same size and resolution. Cut-outs of images for the AR references are shown in Figure 4, with intensity images at the top for each type, range images obtained with the Edge method in the middle and range image obtained with the RefLight method at the bottom. 
Shaggy 1 Cut/Loop Shaggy 2 High/Low Loop
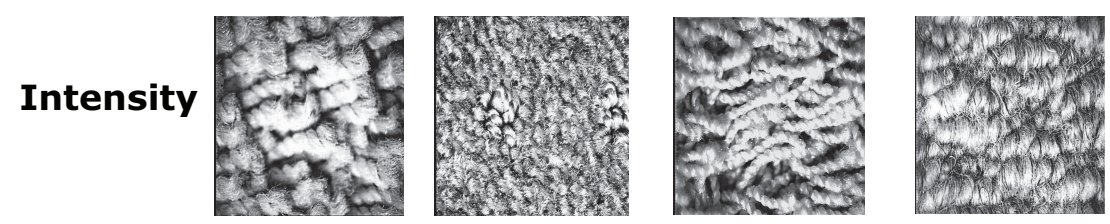

EdgeWav
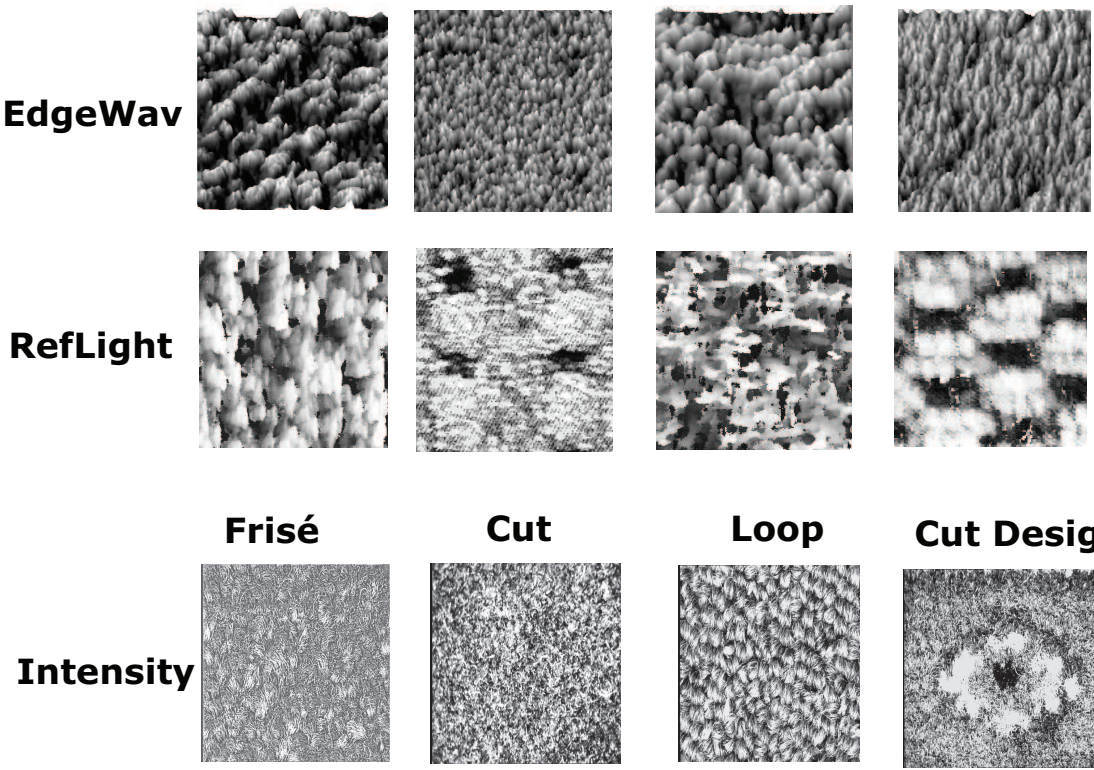

Cut Design
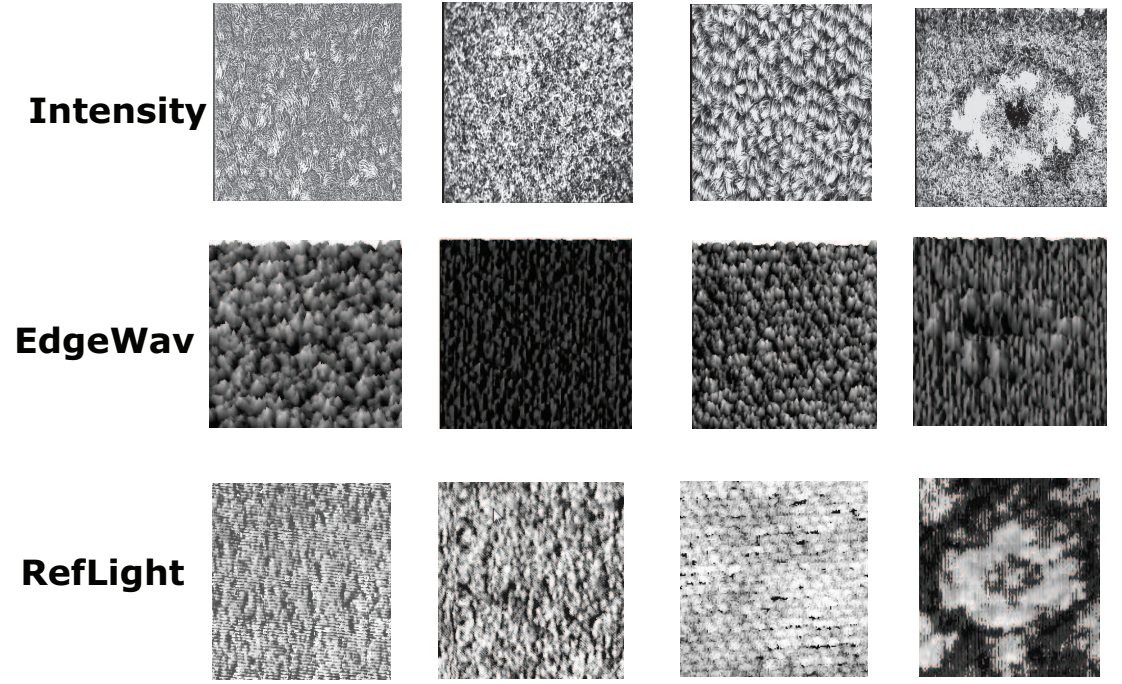

Fig. 4. Intensity and range image samples for the evaluated AR references. 


\subsection{Texture Feature Extraction}

We quantify the difference in texture between images of worn and original appearance by extending a method proposed in a previous approach [13]. The method consists of assigning numbers to the pixels on both images (worn and original texture) by using the Local Binary Pattern (LBP) operator. These numbers represent patterns of local intensity variations. The numbers of occurrences of the patterns along the images are accumulated into LBP histograms, with a single LBP histogram per image. The difference between LBP histograms corresponding to worn and original textures is computed using the Kullback-Leibler divergence (KLD) [32].

Specific bins are discarded in order to enforce that the KLD increases monotonically while the AR grades decrease [26]. The complete method is graphically illustrated in Figure 5 and will be discussed more in detail in the following. We first describe the previous method and then the new component, the removal of bins. 


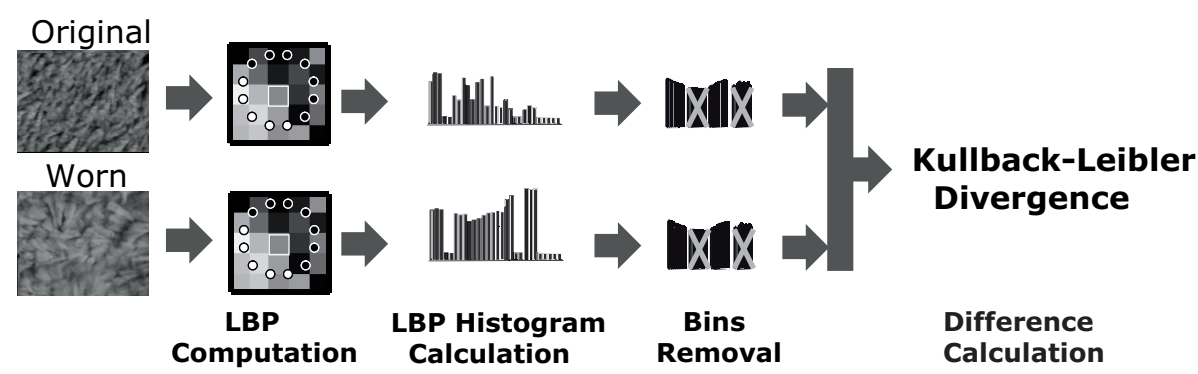

Fig. 5. Computation of texture difference in images of worn and original appearance. 
A) Texture difference measurement. With the aim of leading to an universal automatic system, we have previously proposed a methodology to select optimal image based features for describing AR grades [33]. With this methodology, characteristics from the description between AR grades and the features are quantified and compared using experimental design theory [33]. To select optimal texture features, we use in this investigation the following two quantified characteristics:

- The monotonicity, which measures the order between the KLD values and the AR grades. It is indicated by the symbol $\omega$ within a range from 0 to 1 , which measures, using the Spearman rank correlation, the order of the textures features with the AR grades. $\omega=1$ means perfect order.

- The discriminance, which measures the separability between KLD values of consecutive AR grades. It is indicated by the symbol $\tau$, computed by counting how many times the difference between the average of features corresponding to consecutive AR grades is larger than the threshold for a statistic significance based on the Tukey test [34]. The $\tau$ value is divided into 7 (the number of consecutive pairs of AR grades) to normalize its range from 0 to 1.

Particularly, we have previously compared the performance of the LBP technique against two classical techniques namely, the co-ocurrence matrix technique and the Laws texture energy measures [24]. Results showed that the LBP technique describes more monotonically the transitional texture changes due to wear $(\omega=0.84$ in average for the LBP technique against $\omega=0.61$ and $\omega=0.74$ for co-ocurrence and Laws techniques respectively ) as well as distinguishes a bigger number of consecutive AR grades $(\tau=0.28$ in average for the LBP technique against $\tau=0.42$ and $\tau=0.61$ for co-ocurrence and Laws techniques respectively).

With the LBP technique, we evaluate the neighborhood of a pixel in equidistant points on a concentric circle centered around the pixel [35]. A bit value equal to 1 is assigned to a point on the circle neighborhood of the pixel if the intensity value of the point is bigger than the intensity value of the pixel and a bit value equal to 0 otherwise [36]. One binary code word representing the pattern, is assigned to the pixel by reading out the bin values on the circle neighborhood in a clock wise direction. In this approach, we compute LBP patterns for 8 and 12 circular neighbors using a radius of 1 and $\sqrt{2}$ respectively. Grouping patterns makes the representation becomes 
more compact and invariant to noise. We group mirror, complement and rotational versions of LBP patterns using look up tables [37].

In order to cope with the different types of carpets and textures, it was necessary to identify the best spatial resolution scale that optimally stresses the surface changes for each AR reference. We performed this analysis by keeping the LBP parameters fixed while resizing the images. Images were evaluated at spatial resolution scales within the range from 0.6 to 1 with intervals of 0.1 . Scale factors smaller than 0.6 were not considered because the details of the texture surface vanish at those scales. We automatically selected for each AR reference images with a scale resolution at which the product between $\omega$ and $\tau$ was maximum.

The difference between histograms of LBP is computed using the Symmetric KullbackLeibler (SKL) divergence. One SKL value, called $\kappa$, measures the difference in texture between a pair of images of worn original appearance. We obtain for each type of image within an AR grade a set of $25 \kappa$-values by comparing each other the 5 images of worn against the 5 images of original appearance. Some of the $\kappa$-values from an AR grade can be significantly larger or smaller than the others because of the presence of factors such as flecks or speckles that could not be removed by the vacuum cleaning. These outliers are detected for each AR grade using the Mahalanobis distance between each $\kappa$-value and the whole set of $25 \kappa$-values. The outliers are then replaced with the median from the $\kappa$-values of the corresponding fatigued specimen to allow statistical comparisons with an equal number of texture features per fatigued specimen [34]. Then, we replace a maximum of five outliers to assure valid statistical comparisons between the $\kappa$-values of the AR grades. Using a power analysis test, we calculated that the minimal number of samples required in a set to assure valid statistical comparisons is ten, which means that the $25 \kappa$-values are more than sufficient [34].

\section{B) Bins Removal.}

Detection of most frequently occurring LBPs for particular textures, called dominant LBPs increases the precision in texture classification tasks [38]. Changes of appearance in carpets due to degradation produce fine local texture changes which are not necessarily of dominant texture. Therefore, we are interest in detecting those patterns that change accordingly with the wear. Because the texture due to wear is assumed to change transitionally, we propose to detect in the original LBP histogram the bins of those patterns which frequencies change monotonically. This increases the distinguish of $\kappa$-values corresponding to consecutive AR grades. We called 
this, the Monotonic Local Binary Pattern (MLBP) detection. The bins corresponding to non monotonic LBPs are discarded from the histograms of LBP reducing the histogram dimension [26]. Note that these relevant bins have to be selected for each AR reference.

We identify four types of change in bin behaviors within the original LBP histograms namely $A$ ) bins monotonically decreasing with AR grades, $B$ ) bins with no changes with AR grades $C$ ) bins monotonically increasing with AR grades and $D$ ) bins randomly changing with AR grades. These four types of bin behaviors are illustrated in Figure 6 a). The bins of all AR grades within an AR reference are drawn together to visualize the changes in frequencies of the bins related to the AR grades for each type of carpet. 


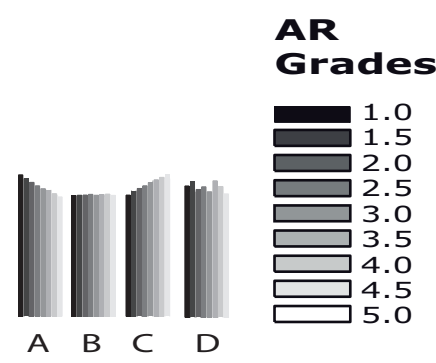

Bin types

a)

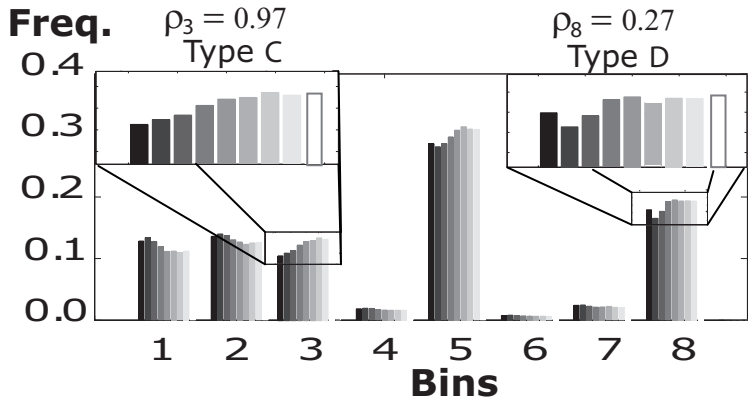

b)

Fig. 6. Detection of LBPs that monotonically change with AR grades. Bins of grades 1.0 to 5.0 are drawn together to visualize the changes in frequencies of the bins related to the AR grades. Types of bin behaviours are shown in Figure a). Figure b) shows an example of the eight first bins out of a total of 125 in images of intensity using patterns with 12 equally spaced circular neighbours in a circle of radius $\sqrt{2}$. Bins 3 and 8 are identified as bins $C$ and $D$ respectively comparing $\varepsilon=0.9$ to their respective $\rho$-values. 
Patterns that monotonically change with the AR grades (Types $A$ and $C$ ) are separated from the others (types $B$ and $D$ ). For this, we compute the Spearman rank correlation, termed $\rho$, between the bin frequencies and the AR grades. the Spearman rank correlation is a measure of statistical dependence between two variables. The absolute value of $\rho$ for each bin is compared to a threshold, termed $\varepsilon$, to remove the non-monotonic patterns ( $B$ and $D$ are those with $|\rho|<\varepsilon$ ) from the LBP histograms. One example of the differentiation of types of bin behaviours ( $C$ and $D$ ) is shown in Figure 6 b). 


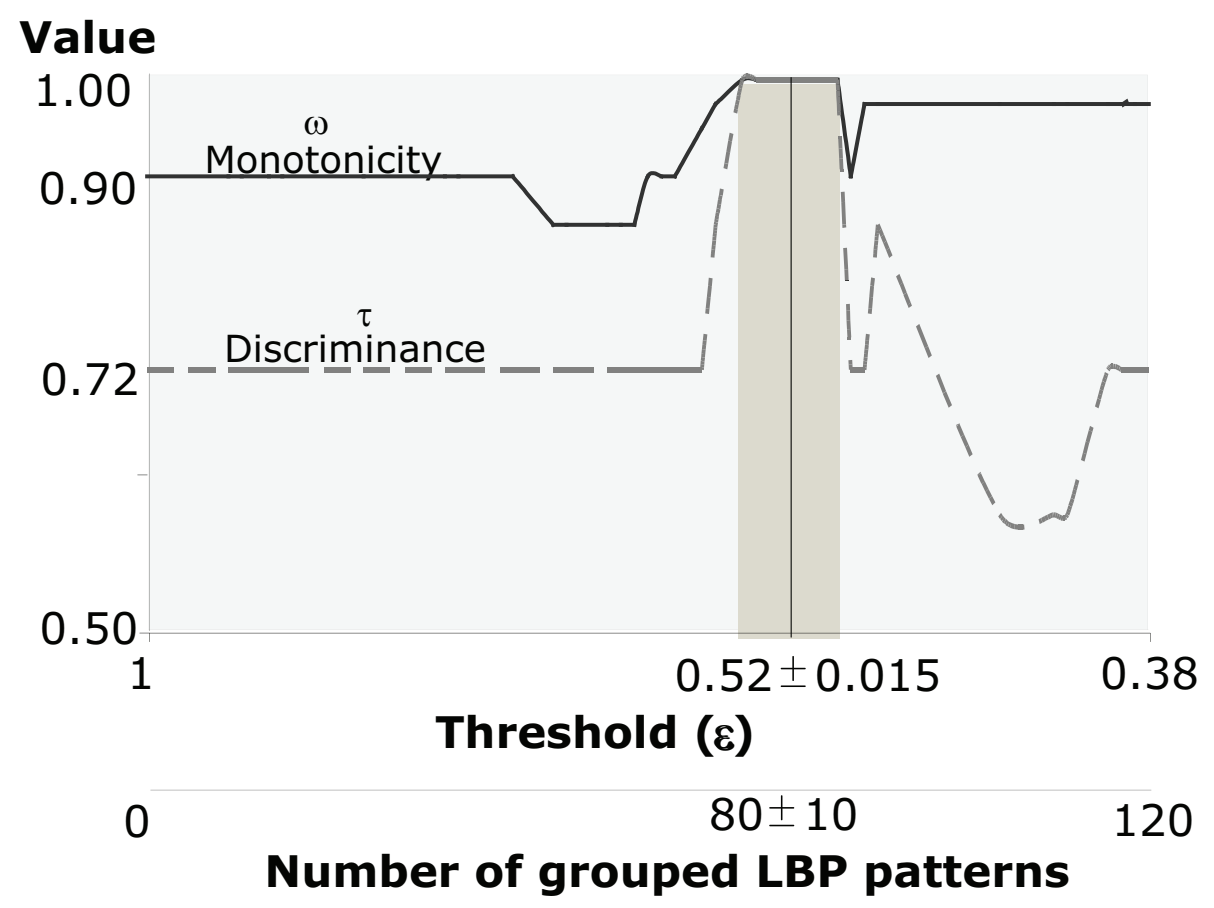

Fig. 7. Example of automatically selecting $\varepsilon$ for carpet type Frisé. In the vertical axis the word value refers to either $\omega$ or $\tau$ in a range from 0 to 1 . The optimal $\varepsilon$ value in this case is found to be in the range given by $0.52 \pm 0.015$, grouping between 70 and 90 LBPs. The rank correlation between $\kappa$-values and AR grades as well as the efficiency discriminating consecutive AR grades are maximum in this range. 
An optimal threshold $(\varepsilon)$ for each AR reference can be obtained by iteratively evaluating, using $\omega$ and $\tau$, the representation of all its $\mathrm{AR}$ grades while decreasing $\varepsilon$ from 1 to 0 . Figure 7 illustrates with one particular example for carpet type Frisé, the common behavior of $\omega$ and $\tau$ when varying $\varepsilon$ in steps of -0.005 . It can be seen that there is a region in which the product of $\omega$ and $\tau$ reaches a maximum value. Therefore, the optimal $\varepsilon$ value can be chosen within this region.

\subsection{Features Combination}

Although the relationship between AR grades and $\kappa$-values is nonlinear, both for depth and intensity images, a linear approximation can be obtained by linearly combining them. This linear description has the benefit that it gives the same probability to each AR grade. We propose to optimally combine the $\kappa$-values using regression models for linearly representing the AR grades [23]. One linear regression model is built for each AR reference. Optimal models are constructed by excluding $\kappa$-values that do not contribute to the variation of the AR grades. In the following we discuss the two steps of the method in detail.

A) Quantification of AR grades using Linear Regression Models. The AR references represent eight different carpet types, which are identified using the index $c=1, \ldots, 8$. The $\kappa$-values are sub grouped according to image type, and the number of circular neighbours. We use $m=1$ for intensity images, $m=2$ for range images using the RefLight method, $m=3$ for range images using the EdgeWav method, $n=1$ for 8 circular neighbors and $n=2$ for 12 circular neighbors. Each of the combinations of image type and number of circular neighbors for an AR reference is called a predictor, denoted by $\kappa_{m n}^{c}$. Subscript $m n$ refers to a combination of image type and number of circular neighbors and superscript $c$ refers to an AR reference. With this notations, the AR grades within an AR reference are linearly modeled using Equation 1.

$$
\hat{W}^{c}=\beta_{0}^{c}+\sum_{m=1}^{3} \sum_{n=1}^{2} \beta_{m n}^{c} \kappa_{m n}^{c}
$$

The corresponding predictors are used together with a set of $\beta$ parameters to estimate the degree of wear of an AR reference, denoted by $\hat{W}^{c}$. Each predictor is associated to one $\beta$ parameter. One extra parameter, termed $\beta_{0}$, is used to correct the bias. The $\beta$-parameters can be trained applying least square methods using the predictors and the AR grades previously 
assessed by human experts. The AR grade for a new fatigued specimen of carpet type $c$, is then automatically computed using Equation 1.

B) Detecting predictors that contribute to AR grading. To identify predictors that do not significantly contribute to the variation of AR grades we use the stepwise regression method [34]. The method computes two statistical measures, namely the adjusted R-squared, denoted by $R_{a}^{2}$, and the Variance Inflation Factor denoted by VIF. Both measures, based on the statistical coefficient of determination $\left(R^{2}\right)$ between the $\kappa$ and $W^{c}$-values.

The $R_{a}^{2}$-value measures the proportion of the variation in $W^{c}$ accounted for by the predictors within a range from 0 to $1 . R_{a}^{2}=1$ means that the AR grades are perfectly described by the linear model. In contrast with the coefficient of determination, the $R_{a}^{2}$-value is independent from the number of predictors.

The $V I F$-value measures the correlations, called multicollinearity, between two subgroups of predictors. If $P$ is the subgroup of predictors evaluated into a linear model and $P^{\prime}$ a subgroup of predictors excluded from the model, the correlation between both subgroups of predictors is denoted by $\operatorname{VIF}\left(P: P^{\prime}\right)$. A multicollinearity problem is detected when the average in terms of the VIF-value, over all possible $P^{\prime} s$, is higher than 5 .

The stepwise regression method iteratively adds the predictor which maximizes $R_{a}^{2}$ without causing a multicollinearity problem.

\section{EXPERIMENT, RESULTS AND DISCUSSIONS}

We have built linear regression models for the eight types of carpets given by the AR references from the EN1471 standard. We captured 30 images from each fatigued specimen representing an AR grade divide into 15 pairs (worn-original) of images. Each of those composed of three subsets of 5 pairs of images ( 5 from photographs, 5 from the RefLight method and 5 from the EdgeWav method). $25 \kappa$-values are computed for each subset of images. We use 15 of the $\kappa$-values per AR grade to construct the models and the other to for validation. In the validation, the AR grades were correctly distinguished and ranked for six of the eight evaluated types of carpets (shaggy 1, loop, cut/loop, high/low loop and frisé). These results are obtained from the following procedure:

We are mainly interested in comparing the performance of $\kappa$-values obtained by combining the LBP and MLBP techniques with the RefLight and EdgeWav methods. To evaluate if features 
extracted using both the RefLight and the EdgeWav methods are complementary estimating the AR grades, we build an additional model evaluating all subgroups of $\kappa$-values. Therefore, we evaluate the following five models, defined as (M-):

1. M-1 $\kappa$-values obtained from LBPs on intensity images and range images using the RefLight method.

2. M-2 $\kappa$-values obtained from LBPs on intensity images and range images using the EdgeWav method.

3. M-3 $\kappa$-values obtained from MLBPs on intensity images and range images using the RefLight method.

4. M-4 $\kappa$-values obtained from MLBPs on intensity images and range images using the EdgeWav method.

5. M-5 $\kappa$-values from all subgroups.

The final $R_{a}^{2}$-values obtained from the stepwise regression method (selecting the best $R_{a^{-}}^{2}$ values with $V I F$ average less than 5) are listed in columns one to four in Table 1 for each carpet. It can be seen from the table that $R_{a}^{2}$-values obtained when using MLBP technique computed on range images of the EdgeWav method perform best among the four models. The $R_{a}^{2}$-values obtained from M-5, are listed in column five of Table 1 for each AR reference. Results show that features extracted using the RefLight method increase the $R_{a}^{2}$-values for some types of AR references. Range images using the RefLight method include information of distorted fibers, which represent the hairiness appearance. Therefore, this result indicates that features quantifying such appearance are complementary for linearity describing the AR changes. 
Table 1. $R_{a}^{2}$-values obtained from the stepwise regression method. The values are listed for each type of AR reference

\begin{tabular}{|c|c|c|c|c|c|}
\hline Carpet Type & M-1 & M-2 & M-3 & M-4 & M-5 \\
\hline Shaggy 1 & 0.850 & 0.877 & 0.911 & 0.913 & 0.918 \\
Cut/loop & 0.292 & 0.857 & 0.493 & 0.864 & 0.916 \\
Shaggy 2 & 0.760 & 0.867 & 0.825 & 0.867 & 0.872 \\
High/Low loop & 0.983 & 0.980 & 0.982 & 0.980 & 0.984 \\
Frisé & 0.909 & 0.930 & 0.933 & 0.943 & 0.954 \\
Cut & 0.961 & 0.961 & 0.961 & 0.961 & 0.961 \\
Loop & 0.934 & 0.939 & 0.905 & 0.939 & 0.939 \\
Cut Design & 0.705 & 0.898 & 0.748 & 0.898 & 0.946 \\
\hline
\end{tabular}


The five models have been validated using the $\kappa$-values from the set of validation containing data from two intensity and two range images per fatigued specimen. To validate, we compute $\kappa$-values combining pairs of range and intensity images from those images resulting in four AR grades per fatigued specimen. The final AR grade of a fatigued specimen is assessed by the average of the four grades.

Figure 8 shows the AR grades results of the linear model of carpet type cut/loop for the combination of $\kappa$-values from one intensity image and one range image. Figure 8 displays in the horizontal axis the AR grades assigned by human experts and in the vertical axis the average of the results of the AR grades obtained with the linear models. It can be seen that in this case, M-5 correctly describes seven of the eight AR grades. 


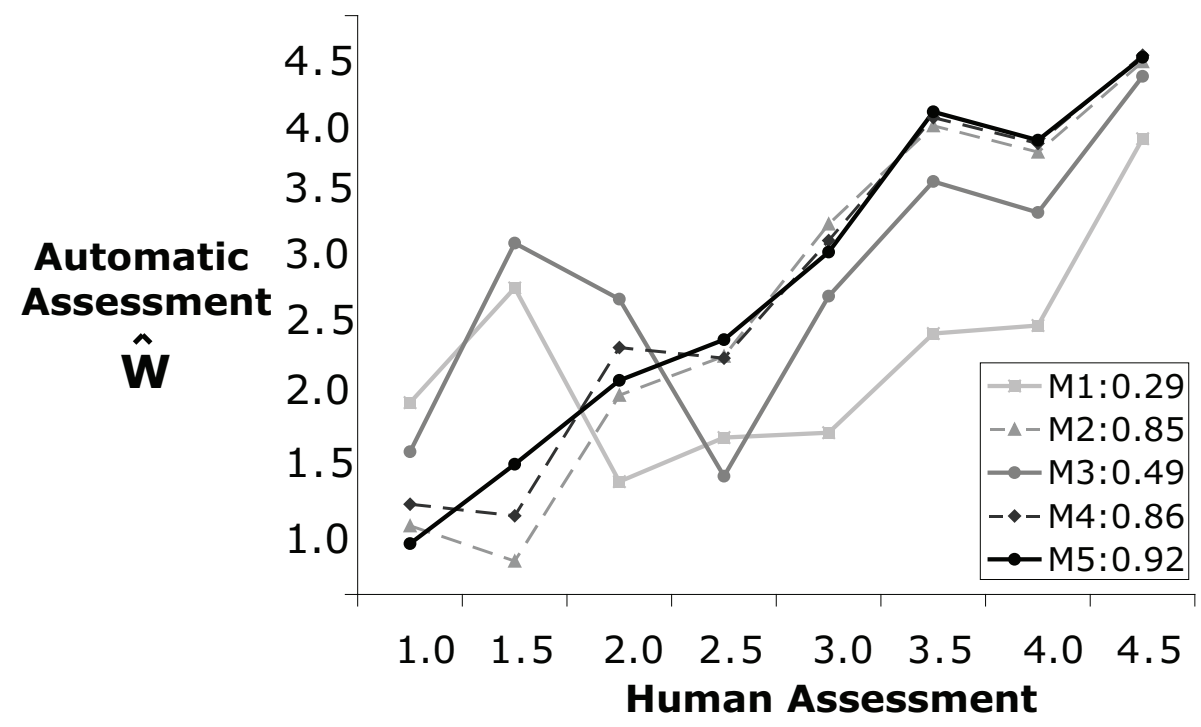

Fig. 8. Validation example for carpet type cut/loop. Models are denoted by M and the corresponding $R_{a}^{2}$-values are shown after ':'. The mean values are connected with lines to visualize the linearity of the models. 
The trend results of the validations for the eight AR references are shown in Figure 9.

The figure shows that for six of the eight AR references, the models adequately describe the estimated degree of wear compared to the human assessment, with grade errors within the 0.5 grade (Identified by points within gray light squares, bigger errors are those points within dark gray squares) that are allowed to human inspectors by international standards. This result is an important step towards the development of an automatic grading system, complying with international standardizations, for evaluating appearance retention in textile floor coverings.

The 3D information captured while scanning the fatigued specimens can be used to quantify several features related to appearance characteristics such as texture, hairiness, structure and thickness. The range images used in this approach are constructed using all information in the surface together to describe the overall texture. Further improvement can be expected by independently computing image features representing separated appearance characteristics. This may be particularly helpful for improving the AR grade representation of AR references, like those in Figure $9 \mathrm{c}$ ) and h), with errors bigger than 0.5 AR grade.

Considering that information related to colors and patterns can be obtained from intensity images, a complete description of the AR of carpets may be achieved by selecting the optimal features using the linear regression method followed in this investigation. Therefore, including the resulting features within the stepwise regression method may lead to a model that more approximately represent the AR grades of the carpets.

The current scanner is a prototype version. Therefore, effort should be strengthened in improving resolution and speed on the scanning process to devise an industrial grading system. The current approach has been performed using limited samples for each AR reference type. Therefore, more robust inferences can be obtained by computing statistics on larger datasets. Particularly, one database for each AR reference type composed of samples better representing the texture diversity present in the market.

We believe that the presented methodology can also be useful for identifying correspondences between appearance and construction characteristics of the AR reference types. This is considering that the carpet types of the AR references are defined by a combination of characteristics in the carpet construction such as pile/surface fiber, type of manufacturer and secondary backing among others. The characterization combined with the knowledge of the human specialists into a more advanced modeling techniques may be useful for designing an universal 
grading system.

The findings of this approach could be useful for other applications where quality inspection of transitional changes is required such as of smoothness or roughness; assessment of fabric wrinkle recovery, piling propensity and smoothness after repeated laundering; assessment of seam appearance; assessment of crease retention and assessment of appearance retention of finished garments. 

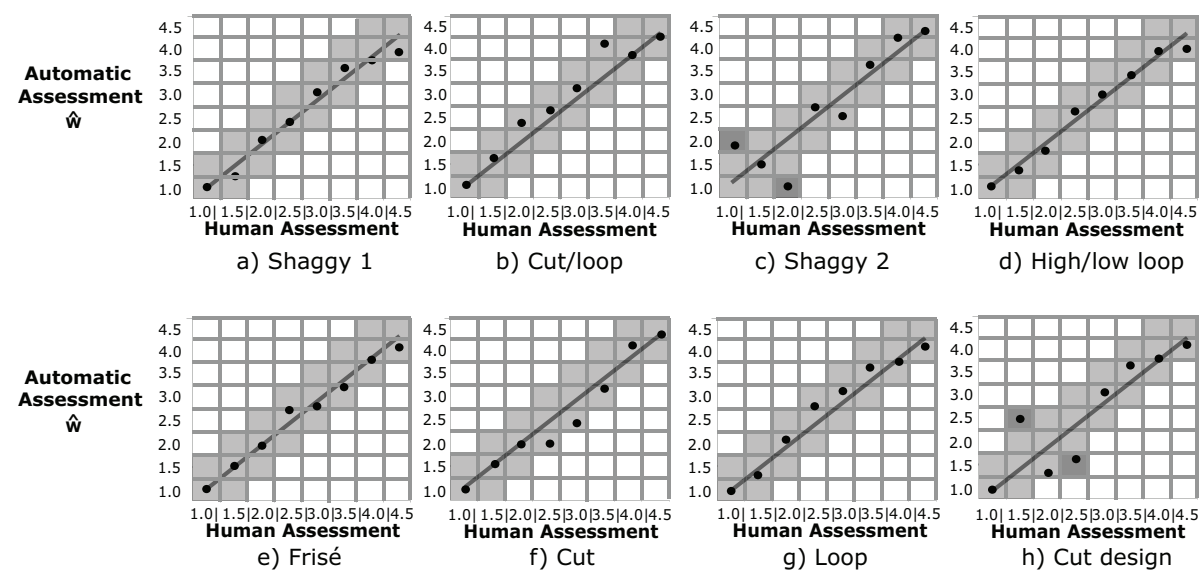

Fig. 9. Validation of the models using $\kappa$-values from two samples of intensity images and two samples of range images. The trends show the results of the AR grade averages obtained with the linear models using two intensity and two range images per fatigued specimen. 


\section{CONCLUSIONS}

In this paper, we have presented an automatic AR grade assessment system for carpets with low pile construction without color patterns, with grade errors within the 0.5 grade allowed by international standards to human inspectors. Therefore, we present a method that is already generic for those types of carpets. This result is a significant step forward for developing an automated assessment system suitable for the requirements of the carpet industry. In carpets with high pile construction the changes were not detected well by our automatic system. These can be attributed to the fact that those changes are related to other surface features such as thickness and hairiness.

With the current system, AR grades are automatically assessed by quantifying the degree of wear in carpets using linear regression models on texture features extracted from intensity and range images obtained with our own carpet scanner. We improved the performance of the automatic system by including range images obtained by detecting edges on the reflected light using the wavelet representation space. Additionally, the dimension of LBP histograms has been optimally reduced by identifying patterns that monotonically change with the AR grades Kullback-Leibler divergences extracted using adjusted histograms offering more relevant information for the linear models. We believe that further studies exploring other kind of features quantifying characteristics such as thickness, hairiness and color patterns, may lead to the development of an automatic AR grading assessment for all types of carpets.

\section{References}

[1] M. Johnston. Carpet specifications and texture retention ratings for commercial carpet. Beaulieu of America, Tek Newsletter, 9(5):3, May 2009.

[2] Textile floor coverings classification of machine-made pile rugs and runners. Textiles Floor Coverings, BS EN 14215:2003, June 2003.

[3] Textile floor coverings - production of changes in appearance by means of vettermann drum and hexapod tumbler testers. Tc 219 Floor coverings, ISO 10361:2000, March 2005.

[4] Constructional details of types of textile floor covering available as reference fatigued specimens. EN1471, 1996. 
[5] L. H. Siew, R. M. Hodgson, and E. J. Wood. Texture measures for carpet wear assessment. IEEE Transactions on Pattern Analysis and Machine Intelligence, 10:92 - 105, 1988.

[6] E. Wood and R. Hofgson. Carpet texture measurement using image analysis. Textile Research Journal, 59:1-12, 1989.

[7] Y. Wu, B. Pourdeyhimi, and S. M. Spivak. Texture evaluation of carpets using image analysis. Textile Research Journal, 61:407-419, July 1991.

[8] J. Sobus, B. Pourdeyhimi, J. Gerde, and Y. Ulcay. Assessing changes in texture periodicity due to appearance loss in carpets: Gray level co-occurrence analysis. Textile Research Journal, 61:557-567, October 1991.

[9] B. Xu. Assessing carpet appearance retention by image analysis. Textile Research Journal, 64:497-509, 1994.

[10] J. Wang and E.J. Wood. A new method for measuring carpet texture change. Textile Research Journal, 65:196-202, April 1994.

[11] S. Sette, L. Boullart, and P. Kiekens. Self-organizing neural nets: A new approach to quality in textiles. Textile Research Journal, 65:196-202, April 1995.

[12] W. Van Steenlandt, D. Collet, S. Sette, P. Bernarn, R. Luning, L. Teze, H. Bohland, and H. Schulz. Automatic assesment of carpet wear using image analysis and neural networks. Textile Research Journal, 66(9):555-561, 1996.

[13] S. A. Orjuela, E. Vansteenkiste, F. Rooms, S. De Meulemeester, R. De Keyser, and W. Philips. Evaluation of the wear label description in carpets by using local binary pattern techniques. Textile Research Journal, 80(20):2132-2143, December 2010.

[14] S. A. Orjuela, E. Vansteenkiste, F. Rooms, S. De Meulemeester, R. De Keyser, and W. Philips. A comparison between intensity and depth images for extracting features related to wear labels in carpets. In Proc. of the SPIE 7798, 2010.

[15] S. A. Orjuela, E. Vansteenkiste, F. Rooms, S. De Meulemeester, R. De Keyser, and W. Philips. Feature extraction of the wear label of carpets by using a novel $3 \mathrm{~d}$ scanner. In Proc. of the Optics, Photonics and Digital Technologies for Multimedia Applications conference, 2010.

[16] W. Waegeman, J. Cottyn, B. Wyns, L. Boullart, B. De Baets, L. Van Langenhove, and J. Detand. Classifying carpets based on laser scanner data. Engineering Applications of Artificial Intelligence, 21(6):907-918, September 2008. 
[17] C. Copot, S. Syafiie, S. A. Orjuela, R. De Keyser, L. Van Langenhove, and C. Lazar. Carpet wear classification based on support vector machine pattern recognition approach. In Proc. of 5th IEEE International conference on Intelligent Computer Communication and Processing, pages 161-164, 2009.

[18] M. J. Karamad, M. Latifi, and M. Amani-Tehran. Nondestructive identification of knot types in hand-made carpet. part I: Feature extraction from grey images. Journal of Nondestructive Evaluation, 28(2):55-62, 2009.

[19] I. H. Sul, K. H. Hong, H. Shim, and T. J. Kang. Surface roughness measurement of nonwovens using three-dimensional profile data. Textile Research Journal, 76(11):828$834,2006$.

[20] K. H. Park, Y. H. Kwon, and K. W. Oh. The surface roughness measurement for textiles fabrics by a non-contact method for tactile perception. In Proc. of the 6th Asian Design Conference, 2003.

[21] D. Semnani, J. Yekrang, and H. Ghayoor. Analysis and measuring surface roughness of nonwovens using machine vision method. World Academy of Science, Engineering and Technology, 57:543-546, 2009.

[22] B. Xin, J. Hu, and G. Baciu. Visualization of textile surface roughness based on silhouette image analysis. Textile Research Journal, 80(2):166-176, 2010.

[23] S. A. Orjuela, E. Vansteenkiste, F. Rooms, S. De Meulemeester, R. De Keyser, and W. Philips. Automated wear label assessment in carpets by using local binary pattern statistics on depth and intensity images. In Proc. of IEEE ANDESCON, pages 1 - 5, 2010.

[24] S. A. Orjuela, E. Vansteenkiste, F. Rooms, S. De Meulemeester, R. De Keyser, and W. Philips. Texture wear analysis in textile floor coverings by using depth information. In Proc. of the XIII Simposio de Tratamiento de señales, Imágenes y Visión Artificial, 2010.

[25] S. A. Orjuela, B. Ortiz, S. De Meulemeester, J. C. Garcia, F. Rooms, A. Pizurica, and W. Philips. Surface reconstruction of wear in carpets by using a wavelet edge detector. In J. Blanc-Talon, D. Bone, W. Philips, Popescu D, and P. Scheunders, editors, Proc. of Advanced Concepts for Intelligence Vision Systems, volume 6474, pages 309-320. Springer, December 2010 . 
[26] S. A. Orjuela, E. Vansteenkiste, F. Rooms, S. De Meulemeester, R. De Keyser, and W. Philips. Analysing wear in carpets by detecting varying local binary patterns. In In Proc. of IS\&T/SPIE Electronic Imaging, 2011.

[27] J. Stachera and P. Rokita. Gpu-based hierarchical texture decompression. In EUROGRAPHICS, 2006.

[28] A. Drimbarean and P. F. Whelan. Experiments in colour texture analysis. Pattern Recognition Letters, 22(10):1161-1167, August 2001.

[29] M. Partio, E. Guldogan, O. Guldogan, and M. Gabbouj. Applying texture and color features to natural image retrieval. In 2003 Finnish Signal Processing Symposium, 2003.

[30] J. Li. A wavelet approach to edge detection. Master's thesis, Sam Houston State University, August 2003.

[31] S. Mallat, and W. L. Hwang. Singularity detection and processing with wavelets. IEEE Trans Information Theory, 38(2):617-614, March 1992.

[32] M. Petrou and P. G. Sevilla. Image Processing Dealing with Texture. Wiley, January 2006.

[33] S. A. Orjuela, R. A. Quinones, B. Ortiz-Jaramillo, F. Rooms, R. De Keyser, and W. Philips. Optimizing feature extraction in image analysis using experimented designs: a case study evaluating texture algorithms for describing appearance retention in carpets. In Mathematics of Data/Image Pattern Coding, Compression and Encryption with Applications XIII, 2011.

[34] M. Kutner, C. J. Nachtsheim, J. Neter, and Li W. Applied Linear Statistical Models. McGraw-Hill/Irwin, 5 edition, 2004.

[35] T. Ojala, M. Pietikäinen, and T. Mäenpää. Multiresolution gray scale and rotation invariant texture classification with local binary patterns. IEEE Transactions on Pattern Analysis and Machine Intelligence, 24(7):971-987, July 2002.

[36] T. Ojala and M. Pietikäinen. A comparative study of texture measures with classification based on feature distributions. Pattern Recognition, 29(1):51-59, January 1996.

[37] S. A. Orjuela, R. Quinones, B. Ortiz-Jaramillo, F. Rooms, R. de Keyser, and W. Philips. Improving texture discrimination in the local binary patterns technique by using symmetry and group theory. In 17th International Conference on Digital Signal Processing, 2011.

[38] S. Liao, W. Max, K. Law, and A. C. S. Chung. Dominant local binary patterns for texture classification. IEEE Transactions on Image Processing, 18(5):1107-1118, 2009. 
Sergio Alejandro Orjuela Vargas graduated as electronic engineer from the National University in Colombia. Afterwards he conducted a postgraduate in statistics in Tolima University. He obtained his a M.Sc. degree in control automation at the University of Ibagué in Colombia in agreement with Ghent and Leuven Universities, Belgium. Since 2008, he is conducting a Ph.D. in Ghent University in the faculty of engineering at the telecommunication department. He is supported by a grant number 20070207 of the 'LASPAU' Academic and Professional Programs for the Americas in agreement with The COLCIENCIAS Science \& Technology Program and Antonio Nariño University, Colombia. His main topic of research is texture analysis. 
Benhur Ortiz-Jaramillo received his bachelor degree in Electronic Engineering from the National University of Colombia, in Manizales, in 2009.

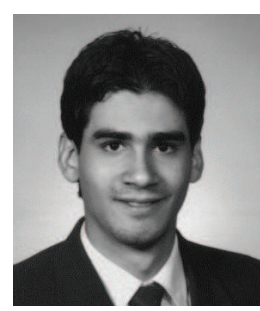

$\mathrm{He}$ is currently a student of the Master program in Industrial Automation of the same university. Since October 2010, he has been doing an internship in Ghent University, Belgium. His main research interests encompass texture feature extraction, digital thermography and their applications to engineering. 
Ewout Vansteenkiste obtained his M.Sc. degree in mathematics in July 2000 and his Ph.D. in image processing in March 2007, both at Ghent University, Belgium. Currently, he is technology developer at the HyCT (Hybrid Computed Tomography) consortium at Ghent University where several teams of Ghent University are working together with industrial partners on innovative technologies for tomographic imaging and image processing for Material Sciences and Life Sciences. 
Filip Rooms obtained his M.Sc. degree in physics at Ghent University, Belgium, in 1999 and his Ph.D. in image processing in 2005 on the topic of image restoration. Afterwards, he worked as a researcher at IncGEO, investigating and implementing software for computer vision in geographical and remote sensing applications. Currently, he has a PostDoc position at Ghent University. 
Simon De Meulemeester finished his studies in 2001 as civil engineer in textiles at Ghent University. He then performed his Ph.D. research on the three dimensional simulation of the weft insertion on air jet looms, finishing in 2010. Besides this, he also worked on several research projects related to air jet looms. An active project he is working on is the development of control strategies for new weft insertion systems. This research occurs in a close cooperation with the loom manufacturer Picanol. He further performed research on the computer based aspect evaluation of carpet wear. 
Robain de Keyser obtained a M.Sc. degree in electro-mechanical engineering in 1974 and the Ph.D. degree in Control Engineering in 1980 from Ghent University, Belgium. He is currently Professor of Control Engineering at the Faculty of Engineering, Ghent University. He acted as external review expert in several European Commission research programs and is one of the pioneers who produced the original concepts of predictive control during the

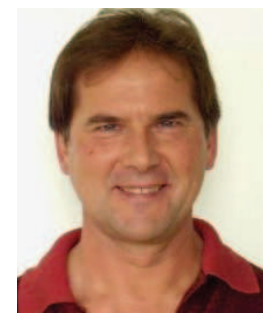
1980s. He has more than 150 papers in peer reviewed journals and international conferences. His research includes many pilot application-driven implementations in technical and non-technical systems, amongst others chemical, steel, marine, mechatronic, semiconductor, power electronics and biomedical. 
Lieva Van Langenhove holds a M.Sc. and a Ph.D. degree in Textile engineering. She is involved in several national and European projects on weaving, smart textiles and biotechnology. She is chairing the ETP expert group on smart textiles and is author of more than 150 papers and presentations at conferences. Furthermore, she is coordinator of the European project SYSTEX, the FP7 coordination action for enhancing the breakthrough of e-textiles and wearable microsystems in Europe. 
Wilfried Philips received the Diploma degree in electrical engineering from the University of Gent, Belgium, in 1989. In 1993 he obtained the Ph.D. degree in electrical engineering from Ghent university, Belgium. He is currently Professor in Ghent University, member of the IPI research group of the department TELIN of the Faculty of Engineering. He has more than 300 papers in peer reviewed journals and international conferences. His main research interests are image and video restoration, image analysis, and lossless and lossy data compression of images and video and processing of multimedia data. 